\title{
Acceptable Incremental Cost Effectiveness Ratio for Use of New Drugs, Cases in Statin Therapies
}

\author{
Susumu Nakao, Hiroyuki Kawabe, Hiroki Takuma, and Makoto Shiragami* \\ Social and Administrative Pharmacy Science Unit, School of Pharmacy, Nihon University, \\ 7-7-1 Narashinodai, Funabashi, Chiba 274-8555, Japan
}

(Received July 30, 2009; Accepted July 6, 2010; Published online July 8, 2010)

\begin{abstract}
As it is an urgent issue to contain increasing healthcare expenditures, unlimited reimbursement of pharmaceuticals continues to be controversial. The objective of this study is to identify acceptable incremental cost effectiveness ratios between new and conventional therapies. Clinical study data for five statin therapies were used to indicate treatment effectiveness and incremental costs were indicated by price premiums at price listing. The incremental cost effectiveness ratios to pravastatin were 0 yen/patient with response, 1,475.1 yen/patient with response, 3,033.3 yen/patient with response, and 3,032.4 yen/patient with response. By conducting further analyses in various pharmaceuticals and categorizing acceptable incremental cost effectiveness ratios based on the disease severity and expected level of improvement in disease condition, drug prices that reflect the value of new pharmaceuticals and that are reasonable to be reimbursed can be suggested.
\end{abstract}

Key words_ _ incremental cost effectiveness ratio; price listing; statin therapy

\section{INTRODUCTION}

Healthcare expenditures in Japan exceeded 3.3 trillion yen in 2005 and continue to increase. Innovation, such as recombinant technology and development of molecular-targeted drugs, has brought about significant advances in treatment of diseases that were considered intractable. At the same time, however, it is also true that such high-priced therapies have placed a burden on the health insurance budget. As it is an urgent issue to contain the increasing healthcare expenditures, rationality for unlimited reimbursement of pharmaceuticals is often discussed in the Central Social Insurance Medical Council and on other occasions. In Japan, new drug prices are determined by the Minister of the Health, Labour and Welfare. In most cases, new drugs are priced by a comparative method, in which daily costs of a new drug is set equivalent to those of a comparator drug, the most similar drug available to the new drug. If the new drug has superiority over the comparator drug, the calculated price is adjusted with price premiums. ${ }^{1}$ The amount of premiums is not set at an absolute value but premium rates are set according to the level of superiority. Therefore, the higher the calculated price is, the premiums become larger and ultimately,

\footnotetext{
*e-mail: shiragami.makoto@nihon-u.ac.jp
}

impact on the healthcare expenditures become greater. Furthermore, when the gap between the calculated price and average price in four overseas countries exceeds a certain level, an upward or downward price adjustment is made.

The value of a pharmaceutical, including not only the costs of the pharmaceutical but also the outcome brought about by the pharmaceutical, may be evaluated by pharmacoeconomic analyses. In order to measure incremental effectiveness of a pharmaceutical, an incremental cost effectiveness ratio between a conventional therapy and the new drug is estimated using the following formula.

Costs of new drug - costs of conventional therapy Effectiveness of new drugs - effectiveness of
conventional therapy

In Western countries, there are attempts to refer to values defined by incremental cost effectiveness ratios in decision making for reimbursement. For examples, Laupacis et al. (1992) in Canada indicated effectiveness of a new therapy by quality adjusted life year (QALY) and suggested that reimbursement is highly reasonable when incremental costs per QALY fall below $\$ 20,000$, while reimbursement is fairly reasonable when incremental costs per QALY are between $\$ 20,000$ and $\$ 100,000$ and is unreasonable when they exceed $\left.\$ 100,000 .{ }^{2}\right)$ In the UK, it was reported that reimbursement is likely to be approved when in- 
cremental costs per QALY below $£ 30,000$ in incremental cost analyses by the National Institute for Health and Clinical Excellence (NICE). ${ }^{3)}$

It may be ideal to describe cost effectiveness by QALY as it enables comparison of values of any technologies. In Japan, however, quality of life (QOL), which is necessary to estimate QALY of therapies, is not often measured in standard clinical studies. It is difficult to have additional evaluation of QOL only for the use of a pharmacoeconomic analysis as it would require extra costs. Furthermore, the concept of QALY is not well understood by those who determine drug prices. It is therefore not realistic to use QALY for this analysis at this stage.

Therefore, thresholds of incremental cost effectiveness ratios to conventional therapies were estimated to provide an indicator for decision making in reimbursement of new pharmaceuticals. In the analysis, treatment effectiveness was indicated by clinical efficacy, which is used in clinical study evaluation, and incremental costs were estimated by price premiums at price listing. The analysis was made in statin therapies, which are used for hyperlipidemia treatment.

\section{MATERIALS AND METHODS}

\section{Cost Effective Analysis}

Effectiveness There are many different endpoints in clinical studies to evaluate effectiveness of drug treatment. In order to estimate acceptable incremental cost effectiveness ratios, which is the objective of this study, it is preferable to use an effectiveness indicator that is common to as many pharmaceuticals as possible. Therefore, the percentage of patients who achieved the treatment target was regarded as an effectiveness indicator. However, as the percentage varies depending on the disease severity, it must be noted that the fact that the percentages are the same does not mean the therapies are of equal value. To estimate effectiveness of statin therapies, clinical study data that were submitted on new drug application and were referred to by the Ministry of Health, Labour and Welfare (MHLW) for drug pricing were used. ${ }^{4-7)}$

In those clinical studies, the endpoint was set at the reduction rate of cholesterol levels from baseline. If the reduction rate is used as incremental effectiveness, an analysis would be made based on the premise that the effectiveness doubles as the reduction rate doubles, which is not appropriate. Therefore, the percentage of patients who achieved the treatment target was estimated based on clinical study data referring to the analytical method used by Wilson et al. (2003) ${ }^{8,9)}$ The details are as follows.

Step 1: Distributions of low density lipoprotein cholesterol (LDL-C) values in the study subjects were estimated for four statin therapies, fluvastatin, atorvastatin, pitavastatin, and rosuvastatin, based on the average baseline LDL-C level in the comparative clinical studies. The values were stratified (hereinafter BAND) by every $5 \mathrm{mg} / \mathrm{dl}$ (Table 1 ). It was assumed that LDL-C values in the study subjects were a population of normal distributions.

Step 2: The LDL-C reduction rate required to achieve the treatment target was calculated for each BAND (Table 1). The treatment target was set at an LDL-C level of $120 \mathrm{mg} / \mathrm{dl}$ according to the 2007 Japan Atherosclerosis Society Guidelines for Diagnosis and Treatment of Atherosclerotic Cardiovascular Diseases, ${ }^{10)}$ which were developed by the Japan Atherosclerosis Society.

Step 3: The number of patients who achieved the treatment target was estimated in the case that each statin therapy was administered to 1,000 patients. The estimation was made as follows. The number of

Table 1. Distribution of Baseline Low Density Lipoprotein Cholesterol (LDL-C) Values in Study Subjects and LDL-C Reduction Rate Required to Achieve Treatment Target

\begin{tabular}{lccccccccccccccccccccccc}
\hline \hline LDL-C value $(\mathrm{mg} / \mathrm{dl})$ & 120 & 125 & 130 & 135 & 140 & 145 & 150 & 155 & 160 & 165 & 170 & 175 & 180 & 185 & 190 & 195 & 200 & 205 & 210 & 215 & 220 & 225 \\
\hline Number of patients & 10 & 13 & 15 & 18 & 21 & 25 & 28 & 31 & 35 & 38 & 41 & 44 & 46 & 47 & 48 & 48 & 47 & 46 & 44 & 42 & 39 & 36 \\
\hline Target reduction rate (\%) & 0 & 4 & 8 & 11 & 14 & 17 & 20 & 23 & 25 & 27 & 29 & 31 & 33 & 35 & 37 & 38 & 40 & 41 & 43 & 44 & 45 & 47
\end{tabular}

\begin{tabular}{lrrrrrrrrrrrrrrrrrrrrr}
\hline \hline LDL-C value $(\mathrm{mg} / \mathrm{dl})$ & 230 & 235 & 240 & 245 & 250 & 255 & 260 & 265 & 270 & 275 & 280 & 285 & 290 & 295 & 300 & 305 & 310 & 315 & 320 & 325 & 330 \\
\hline Number of patients & 32 & 29 & 25 & 22 & 19 & 16 & 13 & 11 & 9 & 7 & 5 & 4 & 3 & 2 & 2 & 1 & 1 & 1 & 0 & 0 & 0 \\
\hline Target reduction rate $(\%)$ & 48 & 49 & 50 & 51 & 52 & 53 & 54 & 55 & 56 & 56 & 57 & 58 & 59 & 59 & 60 & 61 & 61 & 62 & 63 & 63 & 64
\end{tabular}


patients who achieved the treatment target in each BAND of each therapy was calculated based on the LDL-C reduction rate required to achieve the treatment target in each BAND and average LDL-C reduction rate in each therapy. For the average LDL-C reduction rate in each therapy, the average reduction rate after 12 -week treatment in the comparative clinical studies was referred to Table 2. It was assumed that LDL-C reduction rates were normally distributed with the standard deviations in the clinical studies and the same dosages were administered to the patients in all the BANDs. The number of patients who achieved the treatment target was estimated for each therapy by totalling the number of patients who achieved the treatment target in each BAND.

Incremental Costs As the comparator drugs used in drug pricing were the same as the control drugs used in the clinical studies, price premiums at price listing were regarded as incremental costs per day for each therapy (Table 3). As the treatment period in the clinical studies was 12 weeks, costs for the drug treatment were calculated by multiplying the daily incremental costs by 84 ( 7 days $\times 12$ weeks).

Impact on Healthcare Expenditures For pitavastatin and rosuvastatin, an increase in annual healthcare expenditures due to the new drug price listing was estimated based on the market size forecast provided in the document submitted by the pharmaceutical company to the MHLW for drug pricing. The increase in healthcare costs was calculated by multiplying the incremental costs to the comparator drug by the expected number of patients provided in the forecast.

\section{RESULTS}

Estimation of Effectiveness Assuming that in

Table 2. Cholesterol Reduction Rate after 12-week Statin Treatment

\begin{tabular}{lcccc}
\hline \hline \multicolumn{2}{c}{ Drug name } & Dosage & Number of patients & LDL-C reduction rate (\%) \\
\hline Fluvastatin $^{4)}$ & & $30 \mathrm{mg}$ & 192 & $28.0 \pm 15.1$ \\
\hline Pravastatin $^{2}$ Comparator drug & $10 \mathrm{mg}$ & 189 & $22.2 \pm 14.7$ \\
\hline Atorvastatin $^{5)}$ & & $10 \mathrm{mg}$ & 104 & $41.9 \pm 12.5$ \\
\hline Pravastatin $^{*}$ Comparator drug & $10 \mathrm{mg}$ & 108 & $21.5 \pm 13.7$ \\
\hline Pitavastatin $^{6)}$ & & $2 \mathrm{mg}$ & 120 & $37.6 \pm 12.9$ \\
\hline Pravastatin $^{2}$ Comparator drug & $10 \mathrm{mg}$ & 105 & $18.4 \pm 12.0$ \\
\hline Rosuvastatin & & 128 & $40.4 \pm 14.4$ \\
\hline Rosuvastatin & & $5 \mathrm{mg}$ & 128 & $42.9 \pm 14.2$ \\
\hline Atorvastatin & Comparator drug & $10 \mathrm{mg}$ & 127 & $35.1 \pm 14.3$ \\
\hline
\end{tabular}

Table 3. Costs and Number of Patients Who Achieved Treatment Target in 1,000 Patients

\begin{tabular}{|c|c|c|c|c|c|}
\hline \multicolumn{2}{|c|}{ Drug name } & \multirow{2}{*}{$\begin{array}{l}\text { Dosage } \\
30 \mathrm{mg}\end{array}$} & \multirow{2}{*}{$\begin{array}{c}\begin{array}{c}\text { Calculated daily costs } \\
\text { (yen) }\end{array} \\
187.0\end{array}$} & \multirow{2}{*}{$\begin{array}{c}\begin{array}{c}\text { Costs for } 12 \text { weeks } \\
\text { (yen) }\end{array} \\
15,708,000\end{array}$} & \multirow{2}{*}{$\begin{array}{c}\text { Number of patients } \\
328\end{array}$} \\
\hline Fluvastatin & & & & & \\
\hline Pravastatin & Comparator drug & $10 \mathrm{mg}$ & 187.0 & $15,708,000$ & 248 \\
\hline Atorvastatin & & $10 \mathrm{mg}$ & 181.6 & $15,254,400$ & 604 \\
\hline Pravastatin & Comparator drug & $10 \mathrm{mg}$ & 174.4 & $14,649,600$ & 194 \\
\hline Pitavastatin & & $2 \mathrm{mg}$ & 175.2 & $14,716,800$ & 466 \\
\hline Pravastatin & Comparator drug & $10 \mathrm{mg}$ & 163.5 & $13,734,000$ & 142 \\
\hline \multirow[t]{2}{*}{ Rosuvastatin } & & $5 \mathrm{mg}$ & 169.8 & $14,263,200$ & 600 \\
\hline & $\begin{array}{l}\text { After overseas price } \\
\text { adjustment }\end{array}$ & $5 \mathrm{mg}$ & 174.6 & $14,666,400$ & 600 \\
\hline Atorvastatin & Comparator drug & $10 \mathrm{mg}$ & 158.3 & $13,297,200$ & 492 \\
\hline
\end{tabular}


each statin therapy, its comparator drug was administered to 1,000 patients each, the number of patients who were able to achieve the treatment target was estimated (Table 3 ). The number varied depending on the clinical study and ranged from 142 to 248 for pravastatin, which was used in three clinical trials of the clinical studies referred to in this analysis.

Estimation of Incremental Cost Effectiveness Ratios When reduction of the LDL-C value to the target level was regarded as the effectiveness indicator, the incremental cost effectiveness ratio to the comparator drug was the largest in rosuvastatin $(8,944.4$ yen/patient with response) followed by pitavastatin (3,033.3 yen/patient with response) and atorvastatin (1,475.1 yen/patient with response) (Table 4). The incremental cost effectiveness ratio was 0 yen/patient with response for fluvastatin as price premiums had not been given to fluvastatin. For rosuvastatin, an upward price adjustment had been made according to the overseas price adjustment rule. Since cost effectiveness is not reflected in overseas price adjustment, the prices before overseas price adjustment were used in the basic analysis. At the same time, however, it can be considered that the prices after overseas price adjustment are the reimbursement price accepted under the health insurance program. When the price after the adjustment was used in the analysis, the incremental cost effectiveness ratio in- creased to $12,677.8$ yen/patient with response.

Impact on Healthcare Expenditures Introduction of pitavastatin and rosuvastatin to the market caused an increase of approximately 3.6 billion yen and 3.9 billion yen ( 5.6 billion yen after an upward price adjustment), respectively, in the annual healthcare costs (Table 5).

\section{DISCUSSION}

This study was conducted to estimate acceptable incremental cost effectiveness ratios to conventional therapies to provide an indicator for decision making in reimbursement of new pharmaceuticals. In other countries, quality adjusted life year (QALY), which is a common indicator to all treatment methods, is used to indicate effectiveness in similar investigations. Considering methods of standard clinical studies in the country, however, it is not always realistic to use QALY for such analyses. Therefore, the number of patients who achieved the treatment target was estimated based on outcome measurements in clinical studies, and was used as the effectiveness indicator in the analysis. The incremental cost effectiveness ratio was estimated based on price premiums and clinical study data for statin therapies, which are used for hyperlipidemia treatment. This represents costs required for a patient to achieve the treatment target, and in other words, it can be regarded as a value de-

Table 4. Incremental Cost Effectiveness Ratios of Hyperlipidemia Therapies to Comparator Drug in 1,000 Patients

\begin{tabular}{llccc}
\hline \hline Drug name & Comparator & $\begin{array}{c}\text { Incremental effectiveness } \\
\text { (number of patients) }\end{array}$ & $\begin{array}{c}\text { Incremental costs } \\
\text { (yen) }\end{array}$ & $\begin{array}{c}\text { incremental cost effectiveness ratios } \\
\text { (yen/patient with response) }\end{array}$ \\
\hline Fluvastatin & Pravastatin & 80 & 0 & 0 \\
\hline Atorvastatin & Pravastatin & 410 & 604,800 & $1,475.1$ \\
\hline Pitavastatin & Pravastatin & 324 & 982,800 & $3,033.3$ \\
\hline Rosuvastatin & Atorvastatin & 108 & 966,000 & $8,944.4$ \\
\hline Rosuvastatin* & Atorvastatin & 108 & $1,369,200$ & $12,677.8$
\end{tabular}

*After overseas price adjustment.

Table 5. Increases of Healthcare Costs in a Year

\begin{tabular}{|c|c|c|c|c|}
\hline \multicolumn{2}{|c|}{ Drug name } & $\begin{array}{c}\text { Incremental costs } \\
\text { (yen per patient per year) }\end{array}$ & $\begin{array}{l}\text { Estimated number of } \\
\text { patients receiving treatment }\end{array}$ & $\begin{array}{l}\text { Annual incremental } \\
\text { healthcare costs (yen) }\end{array}$ \\
\hline Pitavastatin & & 4,271 & 850,000 & circa. 3.6 billion \\
\hline Rosuvastatin & & 4,198 & 938,000 & circa. 3.9 billion \\
\hline Rosuvastatin & $\begin{array}{l}\text { After overseas price } \\
\text { adjustment }\end{array}$ & 5,950 & 938,000 & circa. 5.6 billion \\
\hline
\end{tabular}


fined by the government to treat a hyperlipidemia patient.

In the analysis of cost effectiveness, effectiveness was indicated by the number of patients who achieved the target for statin treatment. However, in the actual comparative clinical studies, the efficacy was evaluated based on the difference in cholesterol levels between before and after treatment, and thus the number of patients who were able to achieve the treatment target was estimated based on the clinical study results. It is therefore considered that indicating treatment effectiveness by achievement of the treatment target can appropriately reflect a manner in clinical practice.

The more recently the statin therapy was developed, the larger the incremental cost effectiveness ratio became (from 0 to $8,944.4$ yen/patient with response). The incremental cost effectiveness ratio of rosuvastatin was $8,944.4 \mathrm{yen} /$ patient with response to atorvastatin. That of rosuvastatin to pravastatin drawn on a graph of incremental cost and incremental effectiveness was 3,032.4 yen/patient with response, which was almost equal to that of pitavastatin to pravastatin. When these costs are regarded as the upper limit of the acceptable incremental cost effectiveness ratio, it can be said that atorvastatin was priced low (Fig. 1).

In this study, effectiveness of each therapy was estimated based on data for a clinical study, and relative effectiveness of each therapy was estimated based on data for the clinical studies conducted under different conditions. As a result, effectiveness of pravastatin ranged from 142 to 248 . This process may appear imperfect to identify true effectiveness of each therapy.

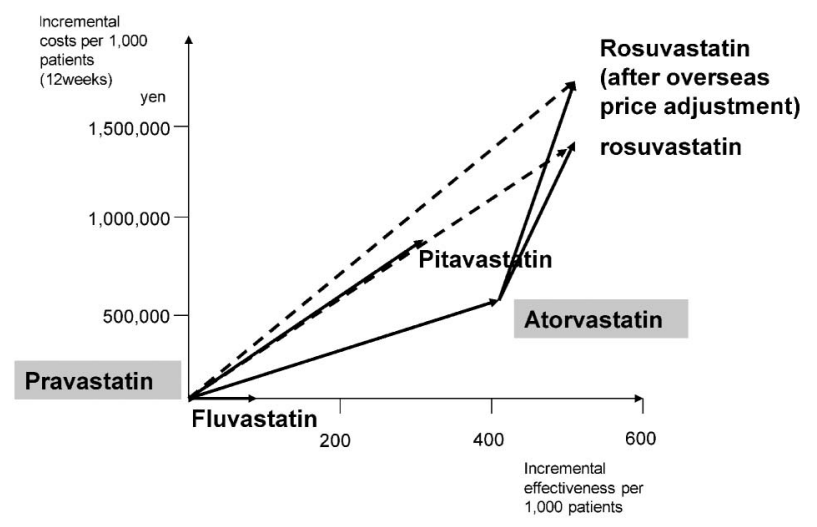

Fig. 1. Incremental Cost Effectiveness Ratios in Statin Therapies
For drug pricing, however, a new drug is evaluated primarily based on data for a clinical study and the results of the evaluation are reflected in price premiums. Considering the objective of this analysis, which was to identify the value recognized for clinical effectiveness of drug treatment, the process of the analysis is thus considered appropriate.

The reasons for using statin therapies for hyperlipidemia treatment in this study included 1) the statin therapies are widely used in the country, 2) treatment of hyperlipidemia has significant impact on the socioeconomy as hyperlipidemia is a chronic condition, and 3) there are more than a few premiumpriced drugs with comparable therapeutic effect. Further analyses should be conducted in various pharmaceuticals in the future, and by categorizing acceptable incremental cost effectiveness ratios based on the disease severity and expected level of improvement in disease condition and identifying acceptable incremental costs per unit, drug prices that reflect the value of new pharmaceuticals and that are reasonable to be reimbursed can be suggested.

Acknowledgments This study was partially funded by a scientific research grant provided by the Japan Society for the Promotion of Science. The Japan Society for the Promotion of Science is an independent administrative institution, established by way of a national law for the purpose of contributing to the advancement of science in all fields of the natural and social sciences and the humanities. The authors have no relevant affiliation or financial involvement with any organization or entity with a financial interest in or a financial conflict with the subject matter or materials discussed in the article.

\section{REFERENCES}

1) Shiragami M., "Health Economics and Policy, Present State and Challenges in Drug Pricing," eds. by Endo H., Ikegami N., Keisoshobo, Tokyo, 2007, pp. 165-184.

2) Laupacis A., Feeny D., Detsky A. S., Tugwell P. X., Can. Med. Assoc. J., 146, 473-481 (1992).

3) Devlin N., Parkin D., Health Econ., 13, 437452 (2004).

4) Fluvastatin Study Group, J. Clin. Thera. Med., 11, 1679-1726 (1995).

5) Kawakami Y., Tagami S., Sakagami S., Prog. 
Med., 18, 2251-2300 (1998).

6) Saito Y., Teramoto T., Yamada N., J. Thera. Med., 17, 857-883 (2001).

7) Pharmaceuticals and Medical Devices Agency: 〈http: // www. info. pmda. go. jp / shinyaku/ g050104 / index.html? submit = \% C9\% BD \% BC\% A8 $>$, PMDA Web, 24 July, 2009.

8) Wilson K., Marriott J., Fuller S., Lacey L., Gillen D., Pharmacoeconomics, 21, 1-11
(2003).

9) Takahashi T., Kamei M., Saegusa Y., Takimoto Y., Shiragami M., Jpn. J. Pharma. Health Care Sci., 32, 320-326 (2006).

10) Japan Atherosclerosis Society, “Japan Atherosclerosis Society Guidelines for Diagnosis and Treatment of Atherosclerotic Cardiovascular Diseases,', 2007 ed., Japan Atherosclerosis Society, Tokyo, 2007, pp. 15-18. 